\title{
JUPITER'S DECAMETRIC AND HECTOMETRIC RADIO EMISSIONS OBSERVED BY CASSINI RPWS AND VOYAGER PRA
}

\author{
M. Imai*i ${ }^{* \dagger}$ A. Lecacheux ${ }^{\ddagger}$, K. Imai ${ }^{\S}$, C. A. Higgins $\mathbb{I}$, and \\ J. R. Thieman"
}

\begin{abstract}
The relationship between Jupiter's decametric (DAM) and hectometric (HOM) radio emissions is important to help understand the emission mechanism that both of them have in common, but it has remained an elusive enigma. We have investigated Jovian DAM and HOM emissions observed by the Cassini, Voyager 1 and Voyager 2 spacecraft. We made a statistical comparison of Cassini and combined Voyager 1 and 2 data for occurrence probability histograms in Central Meridian Longitude (CML) and in Io phase from 2 to $16 \mathrm{MHz}$, and a statistical analysis of Jovian HOM polarization plotted as a function of Jovian magnetic latitude and frequency below $3 \mathrm{MHz}$ based on only the Cassini data. We found that (1) the position of Source B shows shifts in longitude from 10 to $16 \mathrm{MHz}$ as seen in both Cassini and combined Voyager 1 and 2 data, (2) the effect of Io can be seen down to $4 \mathrm{MHz}$, (3) the occurrence probability of HOM emissions are separated into right- and lefthand polarization senses, and (4) attenuation bands make a large contribution to intensify the HOM emissions around the attenuated regions.
\end{abstract}

\section{Introduction}

Although the fact that observations of Jupiter's decametric (DAM) and hectometric $(\mathrm{HOM})$ radio emissions were made from both ground stations and spacecraft since their

* Advanced Course in Mechanical and Electrical Engineering, Kochi National College of Technology, Kochi, Japan

† Now at Department of Geophysics, Kyoto University, Kyoto, Japan

$\ddagger$ LESIA, Observatoire de Paris, UMR CNRS 8109, 92195 Meudon, France

$\S$ Department of Electrical Engineering and Information Science, Kochi National College of Technology, Kochi, Japan

$\mathbb{I}$ Department of Physics and Astronomy, Middle Tennessee State University, Murfreesboro, Tennessee, USA

| Solar System Exploration Division Services Office, NASA Goddard Space Flight Center, Greenbelt, MD 20771 , USA 
discovery, they are still not fully understood, due to their highly complex phenomenology and limited observation conditions (see, Carr et al. [1983], Zarka [2000], Clarke et al. [2004], and references therein]. Since the terrestrial ionosphere limits the ground-based reception of radio waves to approximately $10 \mathrm{MHz}$ and above, only the higher frequency emissions can be usefully studied from the Earth. By contrast, the observable frequency range on spacecraft is limited by on-board receiver capability. So far, only the Planetary Radio Astronomy (PRA) instrument aboard Voyager was designed to fully record the frequency range of both Jupiter's DAM and HOM emissions (up to $40 \mathrm{MHz}$ ), yet it was very difficult to follow the radiation pattern, due to much spacecraft-generated interference concealing observations below $10 \mathrm{MHz}$ and the variance of sensitivity and frequency coverage between high and low frequency receivers. Nevertheless, because Jupiter's HOM and DAM radiation are thought to have a common generation mechanism, it is important to reveal the relationship between them.

In connection with polarization measurements, the PRA aboard Voyager was capable of analyzing the circular polarization senses of the planetary radio emissions, but with incomplete polarization analysis capability. In particular, the apparent sense of circular polarization in continuous emission in time-frequency plot was found to change at around $15 \mathrm{MHz}$. This complicated behavior is due to responses of the antennas with respect to the wavelength, as theoretically studied by Ortega-Molina and Daigne [1984]. Observations by Cassini around Jupiter's flyby provides the opportunity to reexamine this problem. Generally speaking, the short antenna approximation is considered to work only up to about $2 \mathrm{MHz}$ when using 10 meter wire antennas on-board the Cassini spacecraft, but we have applied this technique, which provides useful (but qualitative) polarization profiles, to the higher frequency of the HOM emissions up to $3 \mathrm{MHz}$.

The purpose of this paper is to report the results of a statistical study of the HOM/DAM structure by using the Cassini and Voyager data, providing new information on the statistical analysis of Jovian HOM and DAM radio emissions as well as on the statistical properties of the HOM polarization.

\section{Observations and Analysis}

\subsection{Cassini and Voyager Data}

The Cassini spacecraft was equipped with the Radio and Plasma Wave Science (RPWS) instrument having five on-board receivers that monitored the electric fields from $1 \mathrm{~Hz}$ to $16 \mathrm{MHz}$. The high frequency receiver (HFR) covers the frequency range from $3.5 \mathrm{kHz}$ to $16 \mathrm{MHz}$ and consists of two sets of four analog receivers with a digital signal processing unit. It is connected to three $10 \mathrm{~m}$ long monopole electric field antennas, called here $E_{U}$, $E_{V}$, and $E_{W}$. These are used to receive electric field signals. The $E_{U}$ and $E_{V}$ monopoles are configured at an angle of $120^{\circ}$ and both are nearly orthogonal to the $E_{W}$ monopole. Additionally, the RPWS/HFR is capable of analyzing the polarization responses of radio emissions. For a full description of the RPWS instrument see Gurnett et al. [2004]. For the present study, only the upper bands of HFR (HF1 and HF2) were used. HF1 and 
Table 1: Cassini and Voyager Spacecraft Data Collection Summary

\begin{tabular}{ccccc}
\hline & $\begin{array}{c}\text { Observations } \\
\text { Spacecraft }\end{array}$ & $\begin{array}{c}\text { Spacecraft } \\
\text { distance }\end{array}$ & $\begin{array}{c}\text { Spacecraft } \\
\text { Jovigraphic } \\
\text { latitude }\end{array}$ & $\begin{array}{c}\text { Number of } \\
\text { Jovian rotations } \\
\text { recorded }\end{array}$ \\
\hline Cassini & 2-Oct-00-21-Mar-01 & $1172 R_{J}-1080 R_{J}$ & $+3.7^{\circ}--3.7^{\circ}$ & 339 \\
Voyager 1 & 6-Feb-79-7-Apr-79 & $400 R_{J}-480 R_{J}$ & $+3.2^{\circ}-+5.3^{\circ}$ & 133 \\
Voyager 2 & 8-Jun-79-30-Jul-79 & $356 R_{J}-245 R_{J}$ & $+7.3^{\circ}-+5.2^{\circ}$ & 122 \\
\hline
\end{tabular}

HF2 had a sweep period of 32 seconds over 76 frequency channels, between $0.325 \mathrm{MHz}$ and $4.075 \mathrm{MHz}$ in $50 \mathrm{kHz}$ steps, and 61 channels, from 4.025 to $16.025 \mathrm{MHz}$ every 200 $\mathrm{kHz}$, respectively.

The PRA instrument on the Voyager spacecraft was composed of two on-board receivers that recorded the electric fields from $1.2 \mathrm{kHz}$ to $40.2 \mathrm{MHz}$. The high band receiver is connected to two orthogonal electric antennas with a tip-to-tip length of $10 \mathrm{~m}$, and covers the frequency range from 1.3 to $40.2 \mathrm{MHz}$. It sweeps through 128 channels every 6 seconds with alternating circular polarization from channel to channel and from sweep to sweep, but the 48-second time-averaged data (i.e., 4 averaged LHC and 4 averaged RHC measurements - averaging was done in $\mathrm{dB}$ ), provided by Voyager PRA team, is used here. For a detailed description of the PRA instrument see Warwick et al. [1977].

We have compared the data observed by Cassini, Voyager 1, and Voyager 2. The general geometric parameters used in this study are given in Table 1. Note that, because the geometrical parameters of the spacecraft were rapidly changing near Jupiter's closest approach, we have neglected the data when the Voyager 1 and 2 spacecraft and the Cassini spacecraft were inside of $25 R_{J}$ and $200 R_{J}$, respectively.

\subsection{Statistical Analysis: Rotation Based Threshold}

All the data from Cassini and Voyager are calibrated and normalized to the intensity corresponding to $100 R_{J}$ distance for each frequency channel. The data are organized by Jupiter's rotation. Next, we deduce the mean intensity $(\mu)$ and standard deviation $(\sigma)$ for each rotation of Jupiter. In this study, we define the independent threshold, the mean intensity plus one fifth standard deviation $(\mu+0.2 \sigma)$ for the Cassini case and the mean intensity plus one standard deviation $(\mu+1 \sigma)$ for the Voyager case, since the receiver sensitivity is different for Voyager/PRA and Cassini/RPWS. An activity count occurs when the radio emission intensity is above the threshold, while an observation count occurs if the receiver is operating nominally. The observation and activity counts are sorted into $5^{\circ}$ bins of Jovian CML and $5^{\circ}$ bins of Io phase (in section 3.1), or $0.5^{\circ}$ bins of Jovian magnetic latitude (in section 3.2). Therefore, this technique leads us to compare the strong and weak emissions with the same weight.

The relative occurrence probability is a calculated value between 0 and 1 for each longitude and Io phase bin, or each magnetic latitude bin. The value is just the total number of 
activity counts divided by the total number of observation counts for each bin. Voyager data are simply recorded from a dipole antenna, whereas Cassini data are measured by a three antenna system, $E_{U}, E_{V}$, and $E_{W}$ antennas, so the final occurrence probability of Cassini data is given by the total of the occurrence probabilities for each antenna divided by 3 , the total number of antennas. This is done for each frequency. This technique is more reliable in order to avoid the null effect of any single monopoles for statistical analysis.

\subsection{Polarization Processing on the RPWS/HFR}

Taking into account the basic concept of the polarization analysis on spacecraft presented by Lecacheux [1978] and the methodology of the polarization analysis of the RPWS/HFR performed by Fischer et al. [2009], we have extended the polarization measurements of Jovian HOM emissions below $3 \mathrm{MHz}$ using Cassini data. The way to deduce the polarization response above $2 \mathrm{MHz}$ is based on two main assumptions as follows: (1) the antenna model used is the "equivalent short dipole," which is valid only in the quasi-static approximation for the frequency range below $2 \mathrm{MHz}$ [Vogl et al., 2004]; (2) Jupiter emits only pure circular polarization (i.e., the linear degree Stokes components $Q=U=0$ ), despite the fact that it is known to be false at decametric frequencies. Insofar as we assume the "equivalent short dipole" antenna model, the true circular degree Stokes V is calculated by

$$
V=V_{a} \operatorname{sgn}(\cos (e l)) / \sqrt{1-Q_{a}^{2}-U_{a}^{2}},
$$

where $Q_{a}, U_{a}$, and $V_{a}$ are the measured ("apparent") polarization, and $e l$ is the source elevation above the antenna plane. Because of the neglected antenna gain variation, total intensity $S$ is approximately equal to the apparent intensity $S_{a}$. Hence, right- and left-hand fluxes $S_{R H}$ and $S_{L H}$ are given by

$$
\begin{aligned}
& S_{R H} \approx S(1-V) / 2, \\
& S_{L H} \approx S(1+V) / 2 .
\end{aligned}
$$

Note that the analysis data include the two available antenna pairs $\left(E_{U}, E_{W}\right)$ and $\left(E_{V}\right.$, $\left.E_{W}\right)$, and discard the data where the source direction is too close to being within the antenna plane (i.e., so-called an ill-condition $|\pi / 2-e l|<\pi / 4$ ). In order to determine the occurrence probability, $S, S_{R H}$, and $S_{L H}$ are calculated as described in section 2.2 .

\section{Discussion}

\subsection{CML versus Io Phase}

To analyze the nature of Jupiter's radio emissions, it is generally accepted to make a histogram of Jupiter's radio occurrence probability plotted as a function of either System III CML or the departure angle of the satellite Io from superior geocentric conjunction (Io phase). With regard to the CML plot, there are three significant, well-known zones 

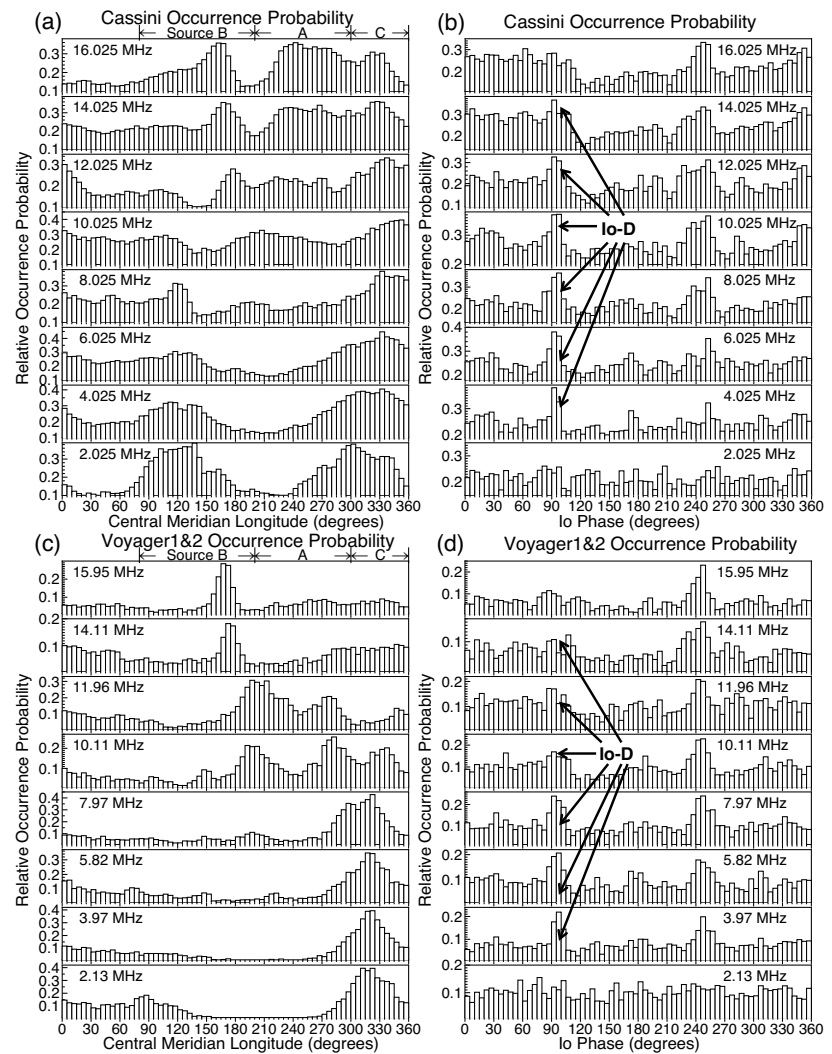

Figure 1: Histograms of occurrence probability based on all Cassini and combined Voyager 1 and 2 data from 2 to $16 \mathrm{MHz}$ in $2 \mathrm{MHz}$ steps. (a) and (c) are plotted as a function of Jovian $C M L$, while (b) and (d) are depicted as a function of Io phase.

in which occurrence probabilities are relatively high. On long-term ground-based observations, above about $18 \mathrm{MHz}$, they are classified as Source B, $80^{\circ} \leq \mathrm{CML} \leq 200^{\circ}$, Source $\mathrm{A}, 200^{\circ} \leq \mathrm{CML} \leq 300^{\circ}$, and Source $\mathrm{C}, 300^{\circ} \leq \mathrm{CML} \leq 360^{\circ}$, in increasing longitude sequence [Carr et al., 1983]. Hereafter we refer to these Sources to explain the lower frequency DAM emissions and the higher frequency HOM emissions.

Figure 1 shows the occurrence probability histograms of Cassini and of combined Voyager 1 and 2 from 2 to $16 \mathrm{MHz}$ in $2 \mathrm{MHz}$ intervals either in System III CML or in Io phase. On comparing the occurrence probability histograms of Cassini with those of Voyager at 14 and $16 \mathrm{MHz}$, there are similar trends (e.g., the peaks of Source B of Cassini and of Voyager at $16 \mathrm{MHz}$ occur at $160^{\circ}$ and $165^{\circ} \mathrm{CML}$ ), but different behaviours of Sources $\mathrm{A}$ and $\mathrm{C}$ are exhibited in Figure 1a and 1c. For instance, at $16 \mathrm{MHz}$, the two peaks of Source A and of Source C appear at $240^{\circ}$ and $320^{\circ}$ in Figure $1 \mathrm{a}$, while no significant peak is detected in Figure 1c. Therefore, our Voyager result suggests that the flux density of Sources A and 


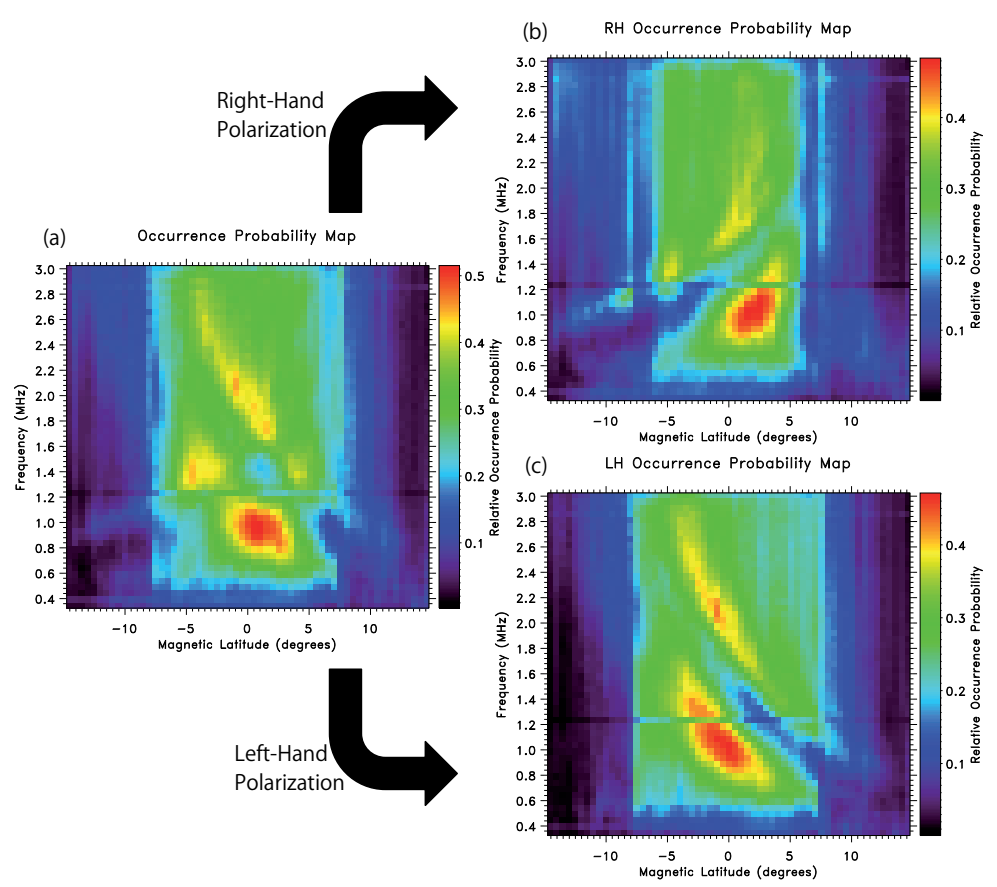

Figure 2: (a) Occurrence probability of HOM from 0.325 to $3.025 \mathrm{MHz}$ is shown as a function of Jovian magnetic latitude. Occurrence probability maps of (b) right- and (c) left-hand polarization are successfully separated from (a) the mixed occurrence probability graph. Note that the attenuated incidence at $1.225 \mathrm{MHz}$ results from continuous interference of the RPWS/HFR.

C are weaker than that of Source B since the sensitivity of the Voyager/PRA receiver is about two orders of magnitude less than that of Cassini/RPWS [Zarka and Kurth, 2005], although we take into account the distance correction.

In Figure 1a, as frequency decreases from $16 \mathrm{MHz}$ down to $10 \mathrm{MHz}$, the peaks of Sources $\mathrm{B}$ and A move toward $205^{\circ}$. At $16 \mathrm{MHz}$, the peaks of Sources B and A occur at $160^{\circ}$ and $240^{\circ}$, but the peaks eventually merge at $10 \mathrm{MHz}$ near $205^{\circ}$. This feature called the "V-shape" was already reported by Imai et al. [2008] and originally found by Thieman and Smith [1978] and Alexander et al. [1981]. In the case of Voyager, in Figure 1c, only Source B is shifted toward $200^{\circ}$ from $16 \mathrm{MHz}$ down to $10 \mathrm{MHz}$. The peak of Source B is shown at $165^{\circ} \mathrm{CML}$ at $16 \mathrm{MHz}$, whereas Source B dominates at $190^{\circ} \mathrm{CML}$ at $10 \mathrm{MHz}$. Below $10 \mathrm{MHz}$, the Voyager occurrence probability histograms show only one region of relatively high occurrence probability, $280^{\circ} \leq \mathrm{CML} \leq 350^{\circ}$ in Figure 1c, while the Cassini histograms show two broad regions, $85^{\circ} \leq \mathrm{CML} \leq 145^{\circ}$ and $290^{\circ} \leq \mathrm{CML} \leq 5^{\circ}$, in Figure 1a.

Concerning the effect of Io, the enhancements of occurrence probability are seen for the Io phase near $90^{\circ}$ and $250^{\circ}$ on the maps above $4 \mathrm{MHz}$ in Figure $1 \mathrm{~b}$ and $1 \mathrm{~d}$. The occurrence probability near $250^{\circ}$ Io phase is relatively high in the frequency range up to $16 \mathrm{MHz}$, 
while the enhancements of incidence near $90^{\circ}$ Io phase can be seen up to $14 \mathrm{MHz}$. This might result from higher occurrence probabilities of the non-Io sources, which appear from $250^{\circ}$ to $100^{\circ}$ (via $360^{\circ}$ ) Io phase in both Cassini and Voyager histograms from 12 to 16 $\mathrm{MHz}$. As an alternative explanation, this might be due to the small, narrow occurrence probability regions of Io- $\mathrm{D}$, one of the prominent Io-related emissions, near $90^{\circ}$ Io phase at $16 \mathrm{MHz}$, in contrast with those below $16 \mathrm{MHz}$, since the duration of Io-D emission in time-frequency plot tends to depend on observed frequency if its series are sorted in the fixed Io phase around $90^{\circ}$. This investigation of Io-related sources will be the subject of future study.

\subsection{Magnetic Latitude versus Frequency}

To further investigate the magnetic latitude dependence of the HOM emissions, we plot in Figure 2 the occurrence probability as a function of the Cassini magnetic latitude and observed frequency. It is important to note that our hypotheses to deduce the polarization responses are not clearly proven by theoretical study, so far considered to work only up to approximately $2 \mathrm{MHz}$ in the case of the RPWS/HFR [Vogl et al., 2004]. Figure 2b and $2 \mathrm{c}$ show the differently polarized structures of the HOM emissions. It is obvious that, as the magnetic latitude increases, the decreased occurrence probability can be seen as the upper frequency drift in Figure $2 \mathrm{~b}$ and the lower frequency drift in Figure 2c, respectively. This decreased occurrence probability corresponds to the attenuation band, or "lane" structure, within HOM radiation [Higgins et al., 1995]. It is well known that this attenuation band tends to change the occurrence profiles plotted by Jovian CML and frequency, but a more reasonable parameter for it is the Jovian magnetic latitude. The separation of the occurrence probability data into spectrograms of RH and LH polarization has not been seen before, and clearly shows different drifts in Figure $2 \mathrm{~b}$ and 2c. These are direct evidence supporting the explanation of this phenomenon produced by ray refraction from a higher plasma density along Io's L-shell [Gurnett et al., 1998; Menietti et al., 2003] since the source of HOM is believed to be $7 \leq \mathrm{L} \leq 11$ [Ladreiter et al., 1994] or $\mathrm{L} \geq 10$ [Menietti et al., 2003]. According to this model, the attenuation bands are the result of the local plasma environment near magnetic flux tubes along Io's L-shell. More importantly, we found that the attenuation bands also intensify the incidence of the HOM emissions around the decreased region (indicated by the red color in Figure 2). We anticipate that this new additional clue would promote a more accurate understanding of the local plasma environment around Jupiter if it was included in analyses using ray-tracing techniques.

\section{Summary}

In this paper we report the occurrence probability histograms of the Cassini and combined Voyager 1 and 2 data from 2 to $16 \mathrm{MHz}$ as well as the statistical analysis of HOM polarization in Cassini data. When the occurrence probability histograms of Cassini data are compared with those of combined Voyager 1 and 2 data, the features of Source $\mathrm{B}$ show similar trends in longitude from 10 to $16 \mathrm{MHz}$, but the different behaviors of Sources $\mathrm{A}$ and $\mathrm{C}$ among these spacecraft observations are also indicated. In addition, 
the HOM structures in the frequency range below $10 \mathrm{MHz}$ are disparate, since they are very sensitive to the parameter of Jovian magnetic latitude. With regard to the effect of Io, both the Cassini and the combined Voyager 1 and 2 occurrence probability histograms clearly show this in the frequency range above $4 \mathrm{MHz}$ around $90^{\circ}$ and $250^{\circ}$ Io phase. Concerning the polarization response of the HOM emissions, we show that there are different behaviors for the right- and left-hand polarization, with different frequency drifts of the attenuation band within HOM emissions on each polarization profile. As a result, the attenuation bands play an important role in not only reducing specific regions of occurrence probability, but also intensifying the occurrence probability of the HOM emissions next to the attenuated regions. This new information suggests a method of indirectly estimating the plasma environment around Jupiter by means of the ray-tracing technique, but this is the subject of a future study.

\section{References}

Alexander, J. K., T. D. Carr, J. R. Thieman, and J. J. Schauble, A. C. Riddle, Synoptic observations of Jupiter's radio emissions: average statistical properties observed by Voyager, J. Geophys. Res., 86, 8529-8545, 1981.

Carr, T.D., M. D. Desch, and J. K. Alexander, Phenomenology of magnetospheric radio emissions, in Physics of the Jovian Magnetosphere, edited by A. J. Dessler, Cambridge University Press, New York, 226-284, 1983.

Clarke, J. T., D. Grodent, S. W. H. Cowley, E. J. Bunce, P. Zarka, J. E. P. Connerney, and T. Satoh, Jupiter's aurora, in Jupiter: the Planet, Satellites, and Magnetosphere, edited by F. Bagenal, W. McKinnon, and T. Dowling, Cambridge University Press, Cambridge, 2004.

Fischer, G., B. Cecconi, L. Lamy, S.-Y. Ye, U. Taubenschuss, W. Macher, P. Zarka, W.S. Kurth, and D. A. Gurnett, Elliptical polarization of Saturn kilometric radiation observed from high latitudes, J. Geophys. Res., 114, A08216, 2009.

Gurnett, D. A., W.S. Kurth, J. D. Menietti, and A. M. Persoon, An unusual rotational modulated attenuation band in the Jovian hectometric radio emission spectrum, Geophys. Res. Lett., 25, 1841, 1998.

Gurnett, D. A., et al. (29 co-authors), The Cassini Radio and Plasma Wave investigation, Space Sci. Rev., 114, 1, 395-463, 2004.

Higgins, C. A., J. L. Green, J. R. Thieman, S. F. Fung, and R. M. Candey, Structure within Jovian hectometric radiation, J. Geophys. Res., 100, 19478, 1995.

Imai, M., K. Imai, C. A. Higgins, and J. R. Thieman, Angular beaming model of Jupiter's decametric radio emissions based on Cassini RPWS data analysis, Geophys. Res. Lett., 35, L17103, 2008.

Ladreiter, H. P., P. Zarka, and A. Lecacheux, Direction finding study of jovian hectometric and broadband kilometric radio emissions: evidence for their auroral origin, Planet. Space Sci., 42, 913-931, 1994. 
Lecacheux, A., Direction Finding of a Radiosource of Unknown Polarization with Short Electric Antennas on a Spacecraft, Astron. Astrophys., 70, 701, 1978.

Menietti, J. D., D. A. Gurnett, G. B. Hospodarsky, C. A. Higgins, W. S. Kurth, and P. Zarka, Modeling radio emission attenuation lanes observed by the Galileo and Cassini spacecraft, Planet. Space Sci., 51, 533-540, 2003.

Ortega-Molina, A., and G. Daigne, Polarization response of two crossed monopoles on a spacecraft, Astron. Astrophys., 130, 301-310, 1984.

Thieman, J. R., and A. G. Smith, Frequency and time dependence of the Jovian decametric radio emissions: A nineteen-year high-resolution study, J. Geophys. Res., 83, 33033309, 1978.

Vogl, D. F., B. Cecconi, W. Macher, P. Zarka, H. P. Ladreiter, P. Fédou, A. Lecacheux, T. Averkamp, G. Fischer, H. O. Rucker, D. A. Gurnett, W. S. Kurth, and G. B. Hospodarsky, In-flight calibration of the Cassini-Radio and Plasma Wave Science (RPWS) antenna system for direction-finding and polarization measurements, J. Geophys. Res., 109, A09S17, 2004.

Warwick, J. W., J. B. Pearce, R. G. Peltzer, and A. C. Riddle, Planetary Radio Astronomy experiment for Voyager missions, Space Sci. Rev., 21, 309-327, 1977.

Zarka, P., Radio emissions from the planets and their moons, in Radio Astronomy at Long Wavelengths, edited by R. G. Stone, J.-L. Bougeret, K. Weiler, and M. Goldstein, American Geophysical Union, Washington, 167-178, 2000.

Zarka, P., and W.S. Kurth, Radio wave emission from the outer planets before Cassini, Space Sci. Rev., 116, 371-397, 2005. 
